\title{
Ultimate gravitational mass defect
}

\author{
O. B. Zaslavskii \\ Department of Mechanics and Mathematics, \\ Kharkov V.N.Karazin National University, \\ Svoboda Square 4, Kharkov 61077, Ukrain *
}

\begin{abstract}
We present a new type of gravitational mass defect in which an infinite amount of matter may be bounded in a zero ADM mass. This interpolates between effects typical of closed worlds and T-spheres. We consider the Tolman model of dust distribution and show that this phenomenon reveals itself for a solution that has no origin on one side but is closed on the other side. The second class of examples corresponds to smooth gluing T-spheres to the portion of the Friedmann-RobertsonWalker solution. The procedure is generalized to combinations of smoothly connected T-spheres, FRW and Schwarzschild metrics. In particular, in this approach a finite T-sphere is obtained that looks for observers in two R-regions as the Schwarzschild metric with two different masses one of which may vanish.

PACS numbers: 04.70.Bw, 04.20 Gz, 04. 20 Jb.
\end{abstract}

\section{INTRODUCTION}

One of the remarkable features of general relativity is a gravitational mass defect because of which a total ADM mass $m$ measured of an external observer at infinity may significantly differ from the proper mass $M$ - total amount of matter contained in the system, $m<M$ 1]. There exist two distinct situations of extremely strong gravitation binding. (i) It can arise in a semi-closed world connected to the external region via "throat". When this throat shrinks, a completely closed world appears separated from the external region, its mass $m$ tending to zero (see monographs [2] - [5] and references therein). (ii) Another type of the gravitation mass defect is inherent to systems without the centre of symmetry that are able

*Electronic address: ozaslav@kharkov.ua 
to accumulate an infinite amount of matter but reveal themselves for an external observer as a body of a finite mass $m[6]$. In particular, this is the property typical of so-called T-spheres 7] - 9] (see below) which in the case of the dust source generalize the Kantowski - Sachs class 10] of exact solutions. The mass $m$ for such models is constant but the T-region can extend infinitely long. (We adhere the classification and terminology introduced in [11]: if the gradient of the areal radius $R$ is space-like, it is called R-region, if it is time-like, it is called T-region. In doing so it is implied that this gradient does not identically vanish.) Being glued to a vacuum region, such a system reveals itself in the outer space as a "Tsphere" of a finite mass. Thus, in the case (i) we are faced with a finite $M$ and zero $m$, in the case (ii) $M$ is infinite, $m$ is finite. It was specially stressed in [9] that the nature of the phenomenon under discussion in these two situations is qualitatively different. The basic aim of the present note is to point out that, nevertheless, both cases can be combined in such a way that they lead to a new type of the gravitational mass defect which is the most possible strong one: an infinite $M$ corresponds to a zero $\mathrm{m}$.

\section{PRELIMINARIES: TOLMAN MODEL AND DEFINITION OF MASS}

For simplicity, we restrict ourselves by perfect dust that admits exact solutions [12]. We begin with short description of their properties. As for dust there exists the frame which is simultaneously synchronous and comoving, the metric under the assumption of spherical symmetry can be cast into the form

$$
d s^{2}=-d \tau^{2}+b^{2}(\chi, \tau) d \chi^{2}+R^{2}(\chi, \tau) d \omega^{2}, d \omega^{2}=d \theta^{2}+d \phi^{2} \sin ^{2} \theta .
$$

Usually, it is implied that the derivative $R^{\prime}$ does not vanish identically (hereafter, prime and dot denote derivatives with respect to $\chi$ and $t$, correspondingly). Then the model admits known Lemaitre-Tolman-Bondi (LBT) family of solutions which describe an inhomogeneous collapse of dust (or its time reversal). However, there is also one more brunch that arises as a special solution of Einstein equations with the areal radius $R=R(t)$ not depending on a spatial coordinate [7], [8], 13]. The special solution under discussion (called "T-spheres" or "T-models" in [7], 8]) possesses a number of unusual properties. Thus, there are two qualitatively different branches of the metric of the type (11). 


\section{A. LBT solutions}

The LBT solutions can be represented in the form

$$
b^{2}=\frac{R^{\prime 2}}{1+f(\chi)},
$$

where $f(\chi)$ should satisfy the inequality $f+1 \geq 0$, otherwise being arbitrary. The time evolution is governed by equation

$$
\dot{R}^{2}=\frac{F(\chi)}{R}+f
$$

the energy density

$$
8 \pi \rho=\frac{F^{\prime}}{R^{\prime} R^{2}},
$$

where $F(\chi)$ is another function parametrizing the solution, $F(\chi)=2 m(\chi)$ (we assume the system of units in which $c=G=1$ ), the quantity $m(\chi)$ is an effective mass introduced first by Lemaitre [14] and later discussed in [15] - [18]. For a generic spherically-symmetrical spacetime it is defined according to

$$
m(\chi)=\frac{R}{2}\left[1-(\nabla R)^{2}\right] .
$$

Its significance consists in that it obeys the simple equation that follows from Einstein equations:

$$
m_{, a}=4 \pi R^{2}\left(T_{a}^{b}-T \delta_{a}^{b}\right) R_{, b},
$$

where $a=0, \chi$ and $T=T_{a}^{a}, T_{\mu}^{\nu}$ is the stress-energy tensor. In the case of dust its only non-vanishing component in the co-moving frame $T_{0}^{0}=-\rho$. It is easy to check that for LTB solutions (21), (3) indeed $m(\chi)=\frac{F(\chi)}{2}$. Eq. (6) with $a=0$ gives in this case $\dot{m}=0$, so that $m(\chi)$ is the integral of motion.

If a configuration of matter (dust in our case) is glued to the vacuum at some $\chi_{0}$, the condition of smoothness requires the continuity of the mass, so that $m\left(\chi_{0}\right)=m$, where $m$ is the ADM mass of the corresponding Schwarzschild solution in the vacuum region [15].

The proper mass is defined according to

$$
M(\chi)=4 \pi \int d l R^{2} \rho=4 \pi \int d \chi b R^{2} \rho,
$$

where $d l$ is the proper distance. One can easy to check that $M(\chi)$ is also an integral of motion in the Tolman model. 
The function $f=f(\chi)$ can have any sign. For our purposes, as will be seen from what follows, it is sufficient to restrict ourselves to the case $f<0$ (so-called elliptic case). Then

$$
\begin{gathered}
R=\frac{F}{2(-f)}(1-\cos \eta), \\
\eta-\sin \eta=\frac{2(-f)^{3 / 2}}{F}\left(\tau-\tau_{0}\right), \tau_{0}=\tau_{0}(\chi),
\end{gathered}
$$

$\tau_{0}$ is the time of a big bang (if $\tau \geq \tau_{0}$ ) or big crunch (if $\tau \leq \tau_{0}$ ).

\section{B. T-spheres}

If $R=R(\tau)$, it is found in [7], 8] that

$$
\begin{gathered}
R=\frac{R_{0}}{2}(1-\cos \eta), \tau=\frac{R_{0}}{2}(\eta-\sin \eta), \\
b=\varepsilon \cot \frac{\eta}{2}+2 M^{\prime}\left(1-\frac{\eta}{2} \cot \frac{\eta}{2}\right), \varepsilon=0, \pm 1,
\end{gathered}
$$

where $R_{0}=$ const. Such a kind of solutions cannot be considered as a particular case of LBT family but, rather, represents a separate branch (nonetheless, T-models can be obtained from a special class of LBT solutions due to a special limiting transition [19]). One can easily

see from (15) that now the quantity $m=\frac{R_{0}}{2}$ is a constant not depending on $\chi$. For a fixed moment of time the spatial geometry represents a hypercylinder.

One more distinctive feature of T-models consists in that they do not have a centre of symmetry and, thus, represent an ideal tool for linking different universes (see, for example, short discussion in recent paper [20]), in this sense being a natural generalization (and materialization) of the Einstein-Rosen bridge.

\section{LBT SOLUTIONS WITH ONE ORIGIN}

Now we demonstrate the example of LTB solutions such that the geometry from the left side approaches that of an infinitely long hypercylinder while from the right side it borders with vacuum and in some limit closes. We will see now that this ensures the ultimate gravitational effect under discussion. Let $f$ near some $\chi=\chi_{0}$ have the asymptotic behavior 


$$
f=-1+k\left(\chi-\chi_{0}\right)^{2}, k>0 .
$$

We also take $F^{\prime}$ to be finite at $\chi_{0}$. Then the proper distance between points with $\chi=\chi_{0}$ and $\chi \neq \chi_{0}$

$$
l=\int d \chi \frac{\left|R^{\prime}\right|}{\sqrt{1+f}}
$$

diverges like $l=-\frac{\left|R^{\prime}\left(\chi_{0}\right)\right|}{\sqrt{k}} \ln \left(\chi-\chi_{0}\right)$ and $\frac{\partial R}{\partial l}=R^{\prime} \frac{\partial \chi}{\partial l} \sim \chi-\chi_{0} \rightarrow 0$. Thus, the coordinate $\chi$ becomes degenerate in this limit, the geometry of manifold tends to that of an infinite hypercylinder, $R=$ const $=R\left(\chi_{0}\right)$. In doing so, the density $\rho \rightarrow \rho\left(\chi_{0}\right)=$ const, so that according to (7) $M$ diverges.

We are still left with the freedom to choose the behavior of functions $f(\chi)$ and $F(\chi)$ in the region $\chi>\chi_{0}$. Let us choose $F(\chi)$ to be monotonically decreasing and vanishing at some $\chi_{1}$. It means that the mass function $m(\chi)$ also vanishes and the world becomes closed from the point of an external observer, its Schwarzschild mass $m=0$. In general, the Tolman model can contain singularities such as surface layers or shell crossing [21], 22], so that we should check that with our choice these singularities do not appear. It follows from the results of the aforementioned works that one may achieve a model with neither surface layer nor shell crossing demanding $F^{\prime}<0, \tau_{0}=0$, provided $\frac{F^{\prime}}{F}-\frac{3}{2} \frac{f^{\prime}}{f}<0$ in the region $\chi_{0} \leq \chi \leq \chi_{1}$. Then $R$ is monotonically decreasing function of $\chi$.

Thus, the above example supplies us with the configuration which is everywhere regular (until a moment of a big crunch or after big bang), the proper mass $M$ is infinite, the mass $m=0$. The geometry of the solution under discussion is such that it has no origin on the left side but has an origin $(R=0)$ on the right side. One can also consider such a profile of the function $m(\chi)$ that at some $\chi_{0}$ it becomes constant: $m=m\left(\chi_{0}\right)>0$ for $\chi \geq \chi_{0}$. Then we will have a semi-closed world that in the limit $m \rightarrow 0$ turns into a closed one.

It is worth noting that in our case inequalities between $m$ and $M$ derived in 23] for any bounded spherically symmetrical distribution of matter do not apply since our system is not bounded and has an infinite proper volume.

\section{PIECEWISE CONSTRUCTIONS}

We will consider now piecewise constructions obtained by gluing between some manifolds corresponding to explicit exact solutions. The general classification of types of smooth gluing 
between different pieces of solutions in the Tolman model was elaborated in [24] but for our purposes it is sufficient to take the simplest examples. Let the matter be described by the Friedmann-Robertson-Walker (FRW) solution in the inner region $\chi \leq \chi_{0}$ with $\chi_{0}>\frac{\pi}{2}$. Outside dust the spacetime is vacuum and is described by the Schwarzschild (S) metric. The FRW and S solutions are easily glued at the boundary surface $\chi=\chi_{0}$ (see [3] for details and references). In doing so, $\tau-\tau_{0}=\int_{R}^{R_{0}} d r\left(\frac{r_{g}}{r}-\frac{r_{g}}{R_{0}}\right)^{-1 / 2}, \tau_{0}$ and $R_{0}$ are some constants, $r_{g}=2 m$ is the gravitational radius, $m$ is the mass measured by an observer at infinity. Inside the matter (see, for example, the problem after Sec. 103 in 1]) the metric takes the form (11) with

$$
b=a(\tau), R=a(\tau) \sin \chi,
$$

where

$$
a=\frac{r_{0}}{2}(1-\cos \eta), \tau=\frac{r_{0}}{2}(\eta-\sin \eta),
$$

$r_{0}$ being the constant, $\chi_{*} \leq \chi \leq \chi_{0}$. For any $\chi_{0}$ the quantity $R\left(\tau, \chi_{0}\right)$ coincides with $R$ calculated according to the outer S metric.

Let the region $\chi \leq \chi_{*}$ correspond to the T-sphere. Thus, the whole sequence of spacetimes (from the left to the right) can be written as T-FRW-S. Gluing T-sphere to the metric (11) along the surface $\chi=\chi_{*}$ produces the extrinsic curvature tensor $K_{\mu}^{\nu}$ whose only non-zero component $K_{2}^{2}=-\frac{R^{\prime}}{R b}$. If $\chi_{*}$ is such that $R^{\prime}=0, R \neq 0$ in the portion of the metric that borders with the T-region, $K_{\mu}^{\nu}$ turns out to be continuous across this surface and, hence, according to general criterion [25] gluing is smooth. Therefore, we may choose the surface $\chi_{*}=\frac{\pi}{2}$ that satisfies this criterion and match the FRW metric to that of the T-model. In doing so, it follows from (10), (15), and (14) that $R$ calculated from both sides (described by the FRW metric and that of the T-sphere) is the same, if we identify the constants $R_{0}=r_{0}$ entering both branches of solutions (LBT ones and T-spheres).

Now we may exploit the facts that (a) smooth gluing demands coincidence of effective masses $m(\chi)$ [15] and (b) the mass $m$ does not depend on $\chi$ for T-models [7]. As a result, for $\chi \leq \frac{\pi}{2} m=$ const $=m\left(\frac{\pi}{2}\right)$. But for FRW solutions the mass $m(\chi)=\frac{r_{0}}{2} \sin ^{3} \chi$, as it follows from (15), (14), (15). Substituting $\chi=\frac{\pi}{2}$, we obtain that $m=\frac{r_{0}}{2}$. The outer observer in the $\mathrm{S}$ asymtotically flat region measures the mass $m\left(\chi_{0}\right)$. In the limiting case $\chi_{0} \rightarrow \pi$ this mass $m\left(\chi_{0}\right) \rightarrow 0$. Meanwhile, as now $\chi$ is not bounded from below and extends infinitely to the region $\chi \leq \frac{\pi}{2}$ corresponding to the T-sphere, the total proper mass $M$ (amount of matter) 
diverges that is typical of T-models [7] - [9]. Thus, gravitational binding is absolute in the sense that an infinite $M$ corresponds to a vanishing $m$.

In the example we have just discussed the T-region extends to minus infinity. However, we may abrupt it and glue smoothly to the left $\mathrm{S}$ metric at some $\chi_{1}$ in the same manner to obtain the model of the type S-T-FRW-S. Repeating all steps we see now that the left observer in its R-region (call it $\mathrm{R}_{2}$ ) of the $\mathrm{S}$ geometry measures the mass $m\left(\chi_{1}\right)=\frac{r_{0}}{2}$, whereas the right one in its R-region (call it $\mathrm{R}_{1}$ ) measures $m\left(\chi_{0}\right)$. In the limit $\chi_{0}=\pi$ the mass $m\left(\chi_{0}\right) \rightarrow 0$ but we want to stress that, in contrast to the case considered in [3] , now the left world separated from the right one is not closed. Moreover, the external left observer measures its nonzero mass. Usually, two Schwarzschildian R-regions do not appear at all when one sews the solution in vacuum with that in matter [4] that occupies some region with $0 \leq \chi \leq \chi_{0}$, with $\chi=0$ corresponding to the centre where $R=0$. However, now this argument does now work since T-region does not have a centre of symmetry at all. It was pointed out in literature [26] that Tolman model admits generalization of the Kruskal construction to the case of non-zero density. The spacetime under discussion which is "made" from known solutions and in which dust is bound between pure vacuum regions, represents another example of such a kind.

Usually, the property $m=0$ and close character of the geometry are considered to be tightly connected and, in quantum theory, this opens the possibility of quantum creation of

such worlds [27] - 29]. However, we saw that in our case the world with a vanishing mass is open from one side and possess an infinite proper volume that seems to be an obstacle to its quantum creation.

\section{CONCLUSIONS}

Within Tolman model, we have manage to find explicit solutions which (i) contain no surface layers and shell crossing, (ii) posses an infinite proper mass and (iii) zero ADM mass. Two kinds of such solutions has been found. The first one is based on LBT solutions only, the second one represents some combinations of LBT solutions and T-spheres. These two cases share the common property: the geometry is essentially different on two sides. On one side, it approaches an infinitely long hypercylinder in LBT case or represents such a hypercylinder precisely in the T-sphere case. On the other side, the geometry is similar to 
that of closed world like in known FRW solutions. In passing, we showed that constructions based on gluing between T-spheres and other Tolman solutions (including pure vacuum ones) lead to asymmetric constructions like two asymptotically flat regions with different masses. In this sense, dust in such solutions represents materialization of the Einstein-Rosen bridge between different universes.

[1] L. D. Landau and E. M. Lifshitz, The Classical Theory of Fields, Pergamon, New York (1975).

[2] B. K. Harrison, K. S. Thorne, M. Wakano and J. A. Wheeler, Gravitation theory and gravitational collapse. Univ. Chicago Press, Chicago 1965).

[3] Ya. B. Zel'dovich and I. D. Novikov, The Structure and Evolution of the Universe (1983) (University of Chicago Press).

[4] Ya. B. Zel'dovich and I. D. Novikov, Stars and Relativity (1971) (University of Chicago Press).

[5] V. P. Frolov and I. D. Novikov, Physics of Black Holes, Kluwer Academic, 1998.

[6] I. D. Novikov, Commun. of the Shternberg State Astronom. Inst. 132, 43 (1964).

[7] V. A. Ruban, Sov. Phys. JETP (Letters) 8, 414 (1968). [Reprinted: Gen. Rel. Grav. 33, 369 (2001).]

[8] V. A. Ruban, Sov. Phys. JETP 29, 1027 (1969). [Reprinted: Gen. Rel. Grav. 33, 375 (2001).]

[9] V. A. Ruban, Zh.Eksp. Teor. Fiziki 85 (1983) 801 [Sov. Phys. JETP 58, 463 (1983)].

[10] R. Kantowski and R. K. Sachs, J. Math. Phys. 7, 443 (1966).

[11] I. D. Novikov, Commun. of the Shternberg State Astronom. Inst. 132, 3 (1964). [Reprinted: Gen. Rel. Grav. 33, 2259 (2001).]

[12] R. C. Tolman, Proc. Nat. Acad. USA 20, 169 (1934).

[13] Datt, B. (1938). Z. Physik 108, 314 [Reprinted: Gen. Rel. Grav. 31, 1619 (1999)].

[14] G. Lemaître, Ann. Soc. Sci. Bruxelles 53, 51 (1933) [Reprinted: Gen. Rel. Grav. 29, 641 (1997)].

[15] C. W. Misner and D. H. Sharp, Phys. Rev. B 136, 571 (1964).

[16] M. A. Podurets, Astron. 41, 28 [Sov. Astr. A. J. 8, 19 (1964)].

[17] V. A. Berezin, V. A. Kuzmin and I. I. Tkachev, Phys. Rev. D 36, 2919 (1987).

[18] E. Poisson and W. Israel, Phys. Rev. D 41, 1796 (1990).

[19] O. B. Zaslavskii, Phys. Rev. D 72 (2005) 067501. 
[20] J. R. Gair, Class. Quant. Grav. 19, 6345 (2002).

[21] C.Hellaby and K. Lake, Astrophys. Journal 290 (1985) 381, Errata ibid. 300 (1986) 461.

[22] C. Hellaby, Some properties of singularities in the Tolman model, Ph. D. Theses, Queen's University at Kingston, Ontario, Canada, 1985.

[23] P. Bizon, E. Malrc and N. Ó. Murchadha, Class. Quant. Grav. 7 (1990) 1953.

[24] N. P. Humphreys, R. Maartens and D. R. Matravers, Regular Spherical Dust Spacetimes, gr-qc/9804023

[25] W. Israel, Nuovo Cimento B 44, 1 (1966); ibid. 48, 463 (1967): C. W. Misner, K. S. Thorne, and J. W. Wheeler, Gravitation (Freeman, San Francisco, 1973), p. 552.

[26] C. Hellaby, Class. Quantum Grav. 4, 635 (1987).

[27] E. P. Tryon, Nature 246, 396 (1973).

[28] P. I. Fomin, Doklady Akad. Nauk Ukr. SSR. 9A, 831 (1975).

[29] A. V. Vilenkin, Phys. Lett. B 117, 25 (1982). 